\title{
Spontaneous Haemoperitoneum in Pregnancy: A Case Report
}

\section{Zubaida Aliyu ${ }^{*}$, Ugochukwu Ekwunife ${ }^{1}$, Olatunbosun Oke $^{2}$, Simon Oladele Isawunmi ${ }^{3}$, Kayode Adetifa ${ }^{3}$, Fatai Kunle Ajayi ${ }^{1}$, Olusegun Akinniranye ${ }^{3}$, Joseph Alozie ${ }^{4}$}

\author{
${ }^{1}$ Department of Obstetrics and Gynaecology, Lagos, Nigeria \\ ${ }^{2}$ Department of Surgery, Lagos, Nigeria \\ ${ }^{3}$ Department of Anaesthesia and Critical Care, Lagos, Nigeria \\ ${ }^{4}$ Emergency Medicine, Lagoon Hospitals, Lagos, Nigeria \\ Email: ^zubaida.aliyu@yahoo.com
}

How to cite this paper: Aliyu, Z., Ekwunife, U., Oke, O., Isawunmi, S.O., Adetifa, K., Ajayi, F.K., Akinniranye, O. and Alozie, J. (2021) Spontaneous Haemoperitoneum in Pregnancy: A Case Report. Open Journal of Obstetrics and Gynecology, 11, 125-130. https://doi.org/10.4236/ojog.2021.112014

Received: January 13, 2021

Accepted: February 22, 2021

Published: February 25, 2021

Copyright $\odot 2021$ by author(s) and Scientific Research Publishing Inc. This work is licensed under the Creative Commons Attribution International License (CC BY 4.0).

http://creativecommons.org/licenses/by/4.0/ Open Access

\begin{abstract}
Introduction: A rare but severe complication of endometriosis during pregnancy is spontaneous hemoperitoneum in pregnancy (SHiP) with life threatening intraabdominal bleeding. We report the case of SHiP in an IVF twin conception with background endometriosis. Case Report: A 31-year-old G1P0, admitted to Lagoon hospitals Ikoyi on $15^{\text {th }}$ May 2019 for severe abdominal pain, dizziness and restlessness at 24 weeks. Examination revealed grossly distended abdomen, with diffuse tenderness and guarding. She was lethargic and pale. A significant drop in hemoglobin to $3.6 \mathrm{~g} / \mathrm{dL}$. A diagnosis of acute abdomen probably SHiP was made. She was moved to the theatre for emergency surgical resuscitation, jointly with a general surgeon. She underwent a midline laparotomy under general anesthesia. She was monitored in the intensive care unit postoperatively. She made remarkable clinical recovery and was discharged home after Fourteen days of inpatient admission. Conclusion: A greater awareness of SHiP and its associated risk factors, such as pelvic endometriosis, may facilitate the diagnosis of this condition and expedite the intervention to improve maternal and fetal outcomes.
\end{abstract}

\section{Keywords}

Hemoperitoneum, Pregnancy, Endometriosis

\section{Introduction}

Spontaneous haemoperitoneum in pregnancy is rare and can occur in the second half of pregnancy, in labour and sometimes in the early postpartum period. It presents with abdominal pain which could be acute or subacute. The di- 
agnosis is difficult and is made retrospectively after laparotomy.

A rare but severe complication of endometriosis during pregnancy is spontaneous hemoperitoneum in pregnancy (SHiP) with life threatening intra-abdominal bleeding. Endometriosis represents one of the main risk factors for SHiP because of the rupture of utero-ovarian vessels or bleeding from endometriosis implants. Because an increased number of patients with severe endometriosis achieve pregnancy thanks to assisted reproduction techniques, physicians should be aware of endometriosis related $\mathrm{SHiP}$ as a cause of acute abdomen during pregnancy. We report the case of a patient with spontaneous haemoperitoneum in an invitro fertilisation twin conception with background endometriosis.

\section{Case Report}

A 31-year-old gravida 1 para 0 woman, was admitted to Lagoon hospitals Ikoyi on the $15^{\text {th }}$ May 2019 for severe abdominal pain, dizziness and restlessness at 24 weeks gestation. Her medical history was significant for appendectomy at 18 years of age, twin invitro fertilization conception and endometriosis. She had undergonelaparoscopy in 2018 for coagulation and excision of endometriosis in the United Kingdom. The patient's main symptom at presentation was severe abdominal pain without any prior possible provoking incident; the pain was constant and diffuse. There was no associated vaginal bleeding, urinary or bowel symptoms. She had presented 2 weeks prior with moderate abdominal pain, she looked well, afebrile and not pale. Abdominal examination was essentially normal and an ultrasound scanrevealed a normal twin pregnancy, di-chorionic, diamniotic and a normal amniotic fluid volume, and the cervical length was 35 $\mathrm{mm}$, the placentae were fundal. Complete blood count, metabolic profile, and urinalysis were performed. The patient's vital signs were normal: blood pressure of 120/70 mmHg, pulse of 90 beats per minute, regular and of good volume. Her respiratory rate was 15 breaths per minute and her chest was clinically clear. Her laboratory results showed hemoglobin of $10.8 \mathrm{~g} / \mathrm{dL}$ with $8.810^{9} / \mathrm{L}$ leukocytes. Her Haemoglobin genotype was AA, Liver function test and urinalysis were normal.

A diagnosis of an acute abdomen in pregnancy was entertained. Abdominopelvic ultrasound scan revealed minimal amounts of free intraperitoneal fluid, no other abnormality was visualized. Twenty-four hours into admission, abdominal pain resolved completely with oral analgesia, and the vital signs remained stable. She was discharged home on request after 48 hours and scheduled for follow-up in a week.

During the follow-up visit, seven days after discharge, she was lethargic and pale with normal pulse and blood pressure. Her Haemoglobin level had dropped to $8.0 \mathrm{~g} / \mathrm{dl}$. She was offered admission to investigate and treat anaemia and also reassess ultrasound scan findings but she declined.

She represented less than 48 hours later with drowsiness, marked pallor, res- 
tlessness and a further drop in the hemoglobin level to $3.6 \mathrm{~g} / \mathrm{dL}$. At this point, a diagnosis of acute abdomen secondary to probably SHiP was made. She was moved to the theatre for emergency surgical resuscitation, jointly with a general surgeon.

The patient underwent a midline laparotomy under general anesthesia. Intraoperatively there was haemoperitoneum of 5 litres. No uterine rupture was seen. The right and left fallopian tubes and ovaries were adherent to the uterine wall. No active bleeding vessel was seen. Because hemostasis was not achievable with the gravid uterus, a hysterotomy was performed. Two female neonates, fresh still births were extracted. They weighed $700 \mathrm{~g}$ and $680 \mathrm{~g}$. After closing the hysterotomy breach, the uterus reduced its volume. Careful adhesiolysis and careful systematic examination of the abdominal organs revealed only endometriotic deposits; the liver and spleen were normal.

There was great difficulty achieving haemostasis from oozing endometriotic spots and a decision was made to pack the pelvic cavity with 14 large abdominal gauze packs. Abdominal wall skin only was closed with titanium staples, leaving the fascia open. She was transferred to the Intensive care unit of the hospital for further resuscitation and critical care. A re-laparotomy was performed after 36 hours. Haemostasis was confirmed following removal of the guaze packs, peritoneal cavity was lavaged with warm saline, and the laparotomy wound closed en-masse.

The patient received a total of 19 units of red blood cells and 15 units of fresh frozen plasma, 8 pools of platelet concentrate and 2 units of cryoprecipitate. She recovered well and was discharged on postoperative day 14 . She has remained stable on subsequent follow-up visits.

\section{Discussion}

Endometriosis is a common gynecologic condition that affects approximately $10 \%$ of females of reproductive age. It is a chronic inflammatory disease defined as the presence of endometrial-like tissue outside the uterine cavity. Endometriosis generally involves the peritoneum, ovaries, and rectovaginal septum but may also involve other abdominal or extra-abdominal sites. Clinically, the symptoms include dysmenorrhea, pelvic pain, deep dyspareunia, and infertility [1] [2] [3]. Endometriosis is associated with an increased risk of early pregnancy complications, such as ectopic pregnancy and miscarriage, but the literature contains few reports of complications linked to severe endometriosis during the third trimester of pregnancy [2]. A rare but potentially catastrophic complication occurring in advanced pregnancy is hemoperitoneum as a result of spontaneous bleeding from blood vessels [1] [2] [3] [4]. During pregnancy, hemoperitoneum caused by spontaneous rupture of uterine vessels is an extremely rare condition but life-threatening complication associated with maternal and perinatal mortality [2] [5]. Published series report maternal mortality ranging from $3.6 \%$ - 49\% [2]. Maternal mortality associated with SHiP 
dropped dramatically during the second half of the $20^{\text {th }}$ century to approximately $4 \%$, but fetal mortality remains high (approximately $31 \%$ ), with $44 \%$ of the deaths attributable to maternal shock [1] [6]. The diagnosis of this condition is difficult and rarely reached without surgery. Laparotomy is often needed to manage these patients. Typical presenting symptoms are sudden intense abdominal pain and signs of hypovolemic shock; a marked drop of hemoglobin level is a frequent finding. The pitfall of SHiP is the differential diagnosis. Placental abruption is the most common preoperative misdiagnosis. Other differential diagnoses include uterine rupture; abdominal pregnancy; ruptured appendix; severe preeclampsia; HELLP (hemolysis, elevated liver enzymes, low platelet count) syndrome; acute fatty liver disease, chorioamnionitis, and hepatic or splenic rupture [2]. Approximately 25 cases of SHiP have been described in the literature during the past 20 years. This condition has been described out of labor (61\%), during labor (18\%), and in the early postpartum period (21\%) [6] [7]. Twenty-nine percent of out-of-labor SHiP cases have been reported to occur before 33 weeks of gestation, $39 \%$ of cases occurred between 33 - 37 weeks of gestation, and $32 \%$ of cases occurred at term. Endometriosis is recognized as the major risk factor for SHiP [1] [2]. Hemoperitoneum in pregnant women with endometriosis can be caused by spontaneous rupture of utero-ovarian vessels or bleeding endometriosis implants. The spontaneous endometriosis-linked rupture of utero-ovarian vessels may be attributed to 3 factors: chronic inflammation caused by endometriosis may cause utero-ovarian vessel leakage [2]; adhesions may create tension on these vessels as the uterus becomes enlarged in pregnancy [1]; decidualization of endometrial foci during pregnancy may cause utero-ovarian vessel perforation [2] [3] [6]. Moreover, venous pressure in the utero-ovarian circulation increases during pregnancy. The venous pressure can be accentuated by physical effort, such as muscular activity, coughing, defecation, coitus, or the pushing phase of labor [2]. Our patient did not report any trigger of abdominal pain. Although in a few cases the origin of bleeding remains unknown even during laparotomy, arteries and superficial veins or varicosities on the posterior surface of the uterus or parametria are often the site of bleeding. The 2009 study by Brosens et al. reviewed all cases of SHiP described since 1987 [7]. The authors noticed that SHiP was associated with endometriosis in $>50 \%$ of cases; the diagnosis of endometriosis was unknown until then in almost half of the affected women. Biopsy samples of the site of bleeding during pregnancy were obtained in 5 cases that revealed endometriosis, characterized by prominent vascularization and decidualization of the lesion. In our patient, the diagnosis of endometriosis was already known from her previous surgery and histology report. Several aspects of treating endometriosis remain controversial: whether surgical treatment for endometriosis should be adopted not only to improve fertility but also to prevent significant pregnancy complications such as SHiP; whether a tailored prenatal follow-up for pregnant women with endometriosis 
should be implemented and how it should be performed (obstetrics and gynecology department visit, ultrasound, magnetic resonance imaging); and whether cesarean section should be performed routinely to prevent irreversible fetal damage when laparotomy is required [2]. Lesions caused by severe endometriosis can lead to significant abdominal bleeding during the third trimester of pregnancy [2]. Bleeding from endometrial implants is an established cause of acute hemoperitoneum in pregnancy, and physicians should keep it in mind in cases of abdominal pain during pregnancy [1]. Prompt intervention may be required in such cases, and an expert surgical team should be involved with adequate availability of blood products and a well-equipped intensive care facility, especially in our African environment [8].

\section{Conclusion}

We believe that in gravid females with a history of endometriosis, severe abdominal pain, and a reduction of hemoglobin, physicians should always suspect SHiP. SHiP is a life-threatening condition for both the mother and the fetus, a prompt diagnosis should lead to prompt treatment. A greater awareness of SHiP and its associated risk factors, such as pelvic endometriosis, may facilitate the diagnosis of this condition and expedite the intervention to improve maternal and fetal outcomes.

\section{Conflicts of Interest}

The authors have no financial or proprietary interest in the subject matter of this article.

\section{References}

[1] Brosens, I., Brosens, J.J., Fusi, L., Al-Sabbagh, M., Kuroda, K. and Benagiano, G. (2012) Risks of Adverse Pregnancy Outcome in Endometriosis. Fertility and Sterility, 98, 30-35.

[2] Katorza, E., Soriano, D., Stockheim, D., et al. (2007) Severe Intraabdominal Bleeding Caused by Endometriotic Lesions during the Third Trimester of Pregnancy. American Journal of Obstetrics \& Gynecology, 197, 501.

[3] Stephansson, O., Kieler, H., Granath, F. and Falconer, H. (2009) Endometriosis, Assisted Reproduction Technology, and Risk of Adverse Pregnancy Outcome. Human Reproduction, 24, 2341-2347.

[4] Falconer, H. (2013) Pregnancy Outcomes in Women with Endometriosis. Seminars in Reproductive Medicine, 31, 178-182.

[5] Aziz, U., Kulkarni, A., Lazic, D. and Cullimore, J.E. (2004) Spontaneous Rupture of the Uterine Vessels in Pregnancy. Obstetrics \& Gynecology, 103, 1089-1091.

[6] Passos, F., Calhaz-Jorge, C. and Graca, L.M. (2008) Endometriosis Is a Possible Risk Factor for Spontaneous Hemoperitoneum in the Third Trimester of Pregnancy. Fertility and Sterility, 8, 251-252.

[7] Brosens, I.A., Fusi, L. and Brosens, J.J. (2009) Endometriosis Is a Risk Factor for Spontaneous Hemoperitoneum during Pregnancy. Fertility and Sterility, 92, 1243-1245. 
[8] Mazrin Nur, M.A., Rohana, I., Hamidah, H., Syauki, H. and Roziana, R. (2017) Idiopathic Spontaneous Haemoperitoneum in Pregnancy. International Journal of Reproduction, Contraception, Obstetrics and Gynecology, 6, 4120-4122. 